\title{
Automatic Train Protection Systems
}

\section{Francesco Flammini*}

Innovation \& Security Engineer, Innovation \& Security, Ansaldo STS, Italy

Vehicles used in railway applications are requested to be more and more performable, reliable and safe. The use of computer based railway control systems is nowadays widespread, as it has been proven to be the most effective, if not the only practicable way to pursue such hard requirements.

\section{Automatic Train Protection Systems}

Automatic Train Protection Systems (ATPS) are used in railway control [1] to supervise train speed against an allowed speed profile which is automatically elaborated y the on-board equipment, on the basis of the information received by the signaling (i.e. ground) subsystem. The on board control system, which is installed in train cockpit, has the aim of guaranteeing the respect of the speed profiles, elaborating the so called "braking curves" in order to allow the train to slow down and brake before any stop signal or emergency condition (Figure 1).

In case of an erroneous or late intervention by the train driver, which interacts with the system by a Man Machine Interface (MMI), the on-board control system automatically commands the braking procedure, directly acting on train-borne apparels via a specific interface, namely the Train Interface Unit (TIU).

The European Railway Traffic Management System / European Train Control System (ERTMS/ETCS) is one of the most widespread international standards for the new railway signaling [2] and control systems. The standard provides the specification for an interoperable ATPS aimed at improving both the safety and the performance of railway lines. ERTMS/ETCS specifies three levels of growing complexity and performance, which can be implemented singularly or together, with the lower levels acting as fall-back systems in case of unavailability of the upper ones. All around the world, several projects based on different levels of ERTMS/ETCS have been developed or are under construction, with ERTMS/ETCS level 2 specification being the most successful. ERTMS/ETCS level 2 is based upon an advanced continuous radio signaling system which uses a special version of the GSM standard, namely the GSM-R, as the most important means of communication between the on-board system and the ground system (Figure 2).

For instance, with reference to braking curve of Figure 1, in ERTMS/ETCS the Target Point is given by the so called Movement Authority (MA) while the Maximum Speed is obtained by the so called Static Speed Profile (SSP). The difference between ERTMS/ETCS level 1 and 2 is substantially given by the means of transmission by which such information is obtained by the train: in level 1 the MA and the SSP are discontinuously obtained via radio from the so called balises, devices physically installed between the track lines and energized by the trains passing over them; in level 2 the same information is continuously transmitted by the ground system via messages using the GSM-R network.

The on-board system logic builds the braking model using MA and SSP information together with physical train data, like per axis weight, length, etc. Then another module checks that train speed is below the thresholds and, if not, commands electrical and/or emergency braking via the TIU (Figure 3).

\section{Computer-Based Interlocking}

A railway interlocking system (IXL) is a safety-critical distributed system used to manage train routes and related signals in a station or line section (which is divided into "blocks"). Its development and verification process must respect international safety norms. Modern IXL systems are computer-based and feature a high number of functional requirements, thus making them very complex. The verification and validation includes process of such systems comprises a set of time-consuming activities (hazard-analysis, code inspection, software testing, etc.).

A computer-based IXL is composed of the following entities:

- A safety-critical centralized elaboration unit (indicated with $\mathrm{CPU}$ ), which is meant to run the control software (processes and configuration);

- A Man Machine Interface (MMI), consisting of a display and a functional keyboard which allows the setting and control of train routes;

- A Communication Computer (CC), used to manage the communication via a Wide Area Network (WAN) with a (distant) central Automation System (AS), also providing remote route management, and possibly adjacent IXL;

- A set of Track Circuits (TC), used to detect if a train is occupying the route;

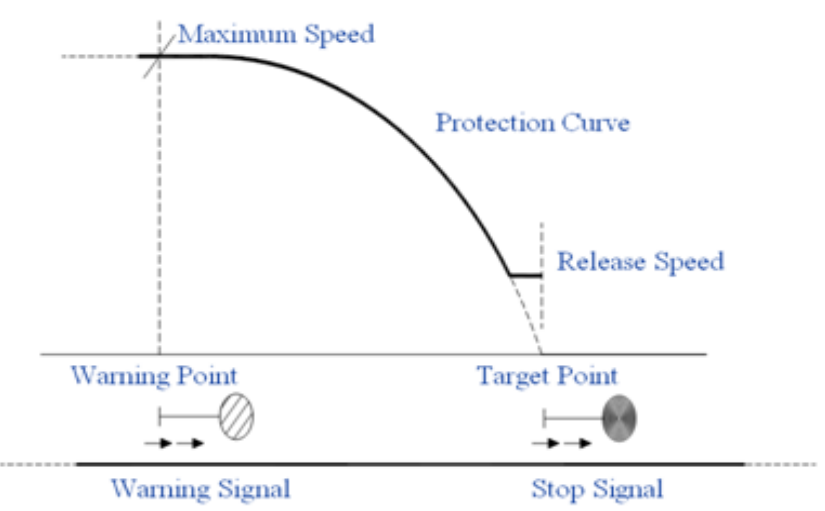

Figure 1: A braking curve or dynamic speed profile.

*Corresponding author: Francesco Flammini*, Innovation \& Security Engineer Innovation \& Security, Ansaldo STS, Italy, Tel: +39 081243 7194; E-mail: frflammi@unina.it

Received September 19, 2013; Accepted November 27, 2013; Published December 02, 2013

Citation: Flammini F (2013) Automatic Train Protection Systems. Ind Eng Manage 2: 120. doi: 10.4172/2169-0316.1000120

Copyright: (c 2013 Flammini F. This is an open-access article distributed under the terms of the Creative Commons Attribution License, which permits unrestricted use, distribution, and reproduction in any medium, provided the original author and source are credited. 


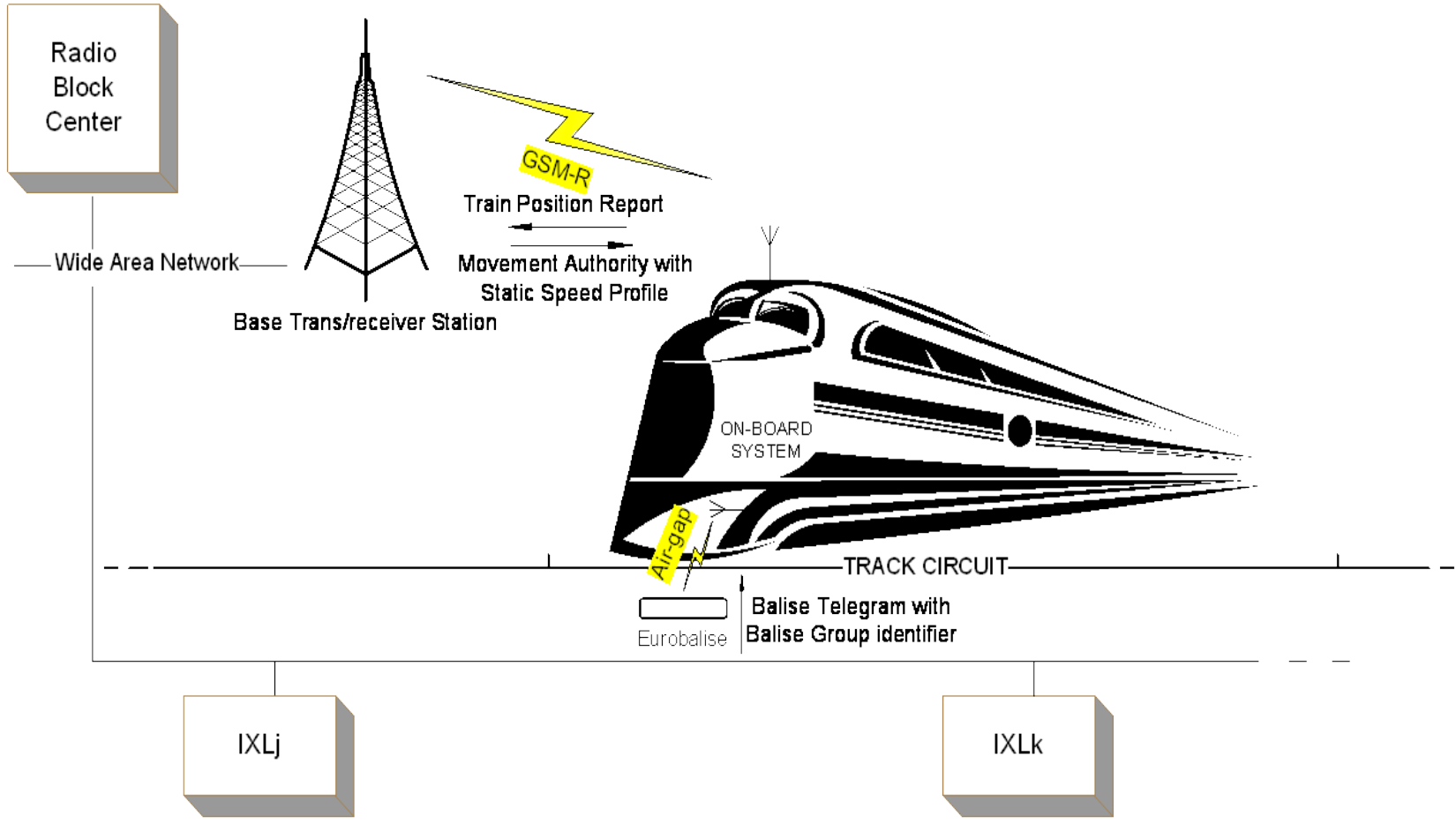

Figure 2: ERTMS/ETCS Level 2 system architecture.

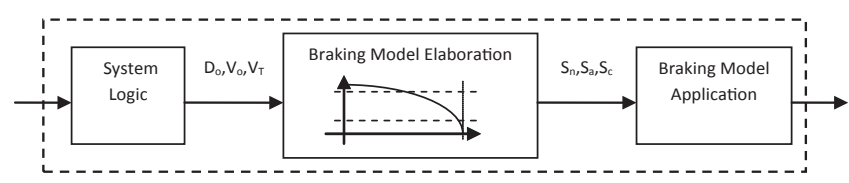

Figure 3: Braking model construction and application

- A set of Switch Points (SP), used to form train route;

- A set of Light Signals (LS), used to notify to train drivers route status.

The IXL configuration associates each possible route to its related physical control entities: TC, SP and LS.

A slightly simplified architecture of an IXL is reported in Figure 4.

An IXL is basically used to manage route formation commands coming from a local human operator (using the MMI) or a remote operator (using the AS). When a command is received, the CPU controls its actability by checking the status of all involved entities, either physical (TC, SP and LS) or logical (e.g. block orientation, line out of service, station emergency, etc.). If the route formation command can be safely carried out, then Switch Points are moved accordingly. A route can also be formed in a degraded mode, in which route integrity cannot be assured because a check failed on a Track Circuit due to its being occupied, or because a Switch Point is not operational. These degradations reflect on route integrity status, which have to be properly notified by multi-aspect Light Signals [3]. Moreover, the system has also to manage the change of route status when a train passes on it, until the liberation of the route. The state machine associated to a route is quite complex: for the sake of simplicity, it will not be described in detail.
First of all, it is important to distinguish between sensor and actuator entities in an IXL. Clearly, Track Circuits can be considered as Sensors, as they are only used to detect train position. Switch Points and Light Signals are instead Actuators, because they are responsible for system control actions. The interface for route setting and monitoring, finally, can be considered both as a Sensor, as it receives commands, and an Actuator, as it displays outputs; analogously for the WAN interface.

In the following, a possible IXL software reference architecture using an object oriented design is provided. Control processes are associated to each physical control entity (TC, SP, LS), thus obtaining:

- TC Process, dealing with Track Circuit status (clear, occupied, broken, etc.);

- SP Process, managing Switch Point status (straight, reverse, moving, out of control, etc.) and operations (move straight, move reverse, etc.);

- LS Process aimed at controlling Light Signals' status (green, red, yellow, flashing yellow, etc.)

Furthermore, logic processes are defined for each logical control entity, thus obtaining:

- Route Process, managing route status and control actions;

- Line Process, managing out of service conditions, temporary speed restrictions, etc.;

- Block Process, managing logical block orientation;

- LeftIXL Process (managing data received by left adjacent IXL) etc.

Also Sensor type processes, if implemented according to object orientation, shall feature the operations needed to access the status of their attributes. 
(a)

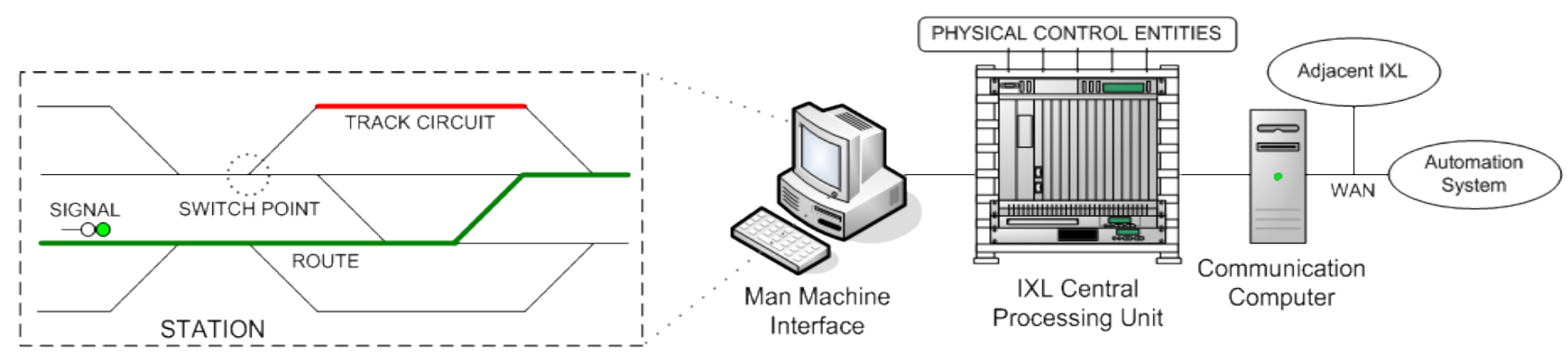

(b)

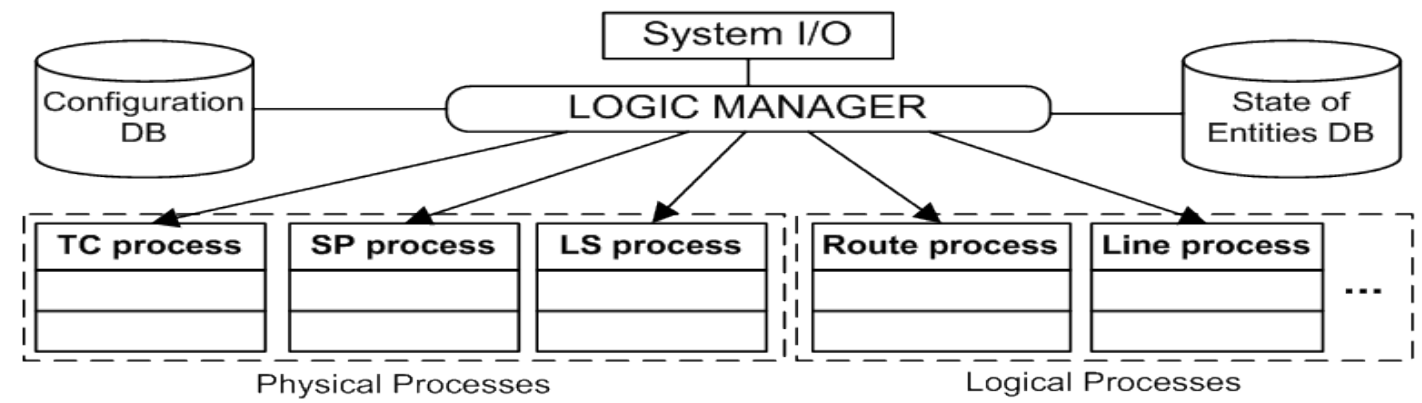

Figure 4: An IXL scheme (a) and related control software architecture (b).

Therefore, an IXL features a real-time kernel scheduling the above mentioned processes. A Logic Manager is usually employed to interpret and schedule processes written in application specific logic languages (Figure $4 \mathrm{~b}$ ). Moreover, a separate and well defined system configuration allows customizing of the IXL to each specific installation (i.e. railway station).

\section{References}

1. Jörn Pachl (2002) Railway Operation and Control. (2nd Edition), Vtd Rail Pub.

2. Brian Solomon (2010) Railroad Signaling. Voyageur Press.

3. Francesco Flammini (2012) Railway Safety, Reliability and Security: Technologies and Systems Engineering. IGI Global. 